\section{Morphological features \\ of secondary lung tuberculosis in HIV-patients}

10.52739/bio-up.1.2021.36-40

Petro V. Kuzyk', Andriy Yu. Horoshchak2, Sofiii
Shrestha', Ludwika Fortuna', Sergiy G. Gychka

\section{ABSTRACT:}

HIV infection) are characterized not only by a high level of

epidemiological indicators, but also by profound changes in the pathogens themselves (multiple drug resistance, etc.), changes in the course of diseases, its' complications, causes of death, clinical and morphological manifestations, the appearance of co-infections, which is regarded as a negative phase of pathomorphosis or as reversion. At the same time the pathomorphosis of diseases significantly complicates their diagnosis. There are objective reasons that do not allow to verify certain nosological units. Under conditions of nosomorphosis due to changes in the biological properties of the pathogen and properties of the ongen an reactive properties of the organism, in clinical practice and in the study of sectional, surgical and biopsy materials often have difficulties in diagnosis of various forms of tuberculosis and HIV patho-, morpho- and thanatogenesis. Untimely diagnosis often leads to disability and mortality of patients. Therefore, in clinica practice, undiagnosed forms of socially dangerous diseases are more common.

In 2018 251,000 people who had both TB and HIV are estimated to have died [1]. This is in addition to the 1.2 million people who

Bogomolets National Medical University, Department of Pathologica

anatomy \#2, Kyiv, Ukraine
Kyiv Medical University, Department of Pathological Anatomy, Histology and Forensic Medicine, Kyiv, Ukraine died from TB alone. A total of 477,461 TB cases among HIV positive people were reported in 2018 (56\% of the estimated incidence of 862,000 cases) [2]. Of these $86 \%$ were on antiretroviral therapy. In comparison, in Ukraine in 20195943 HIV deaths were reported, 1448 among them had TB and HIV coinfection (24.36\%) and 811 of them were on antiretroviral therapy (56\%).

Due to all these statistics we can make a conclusion that TB in HIV patients is one the main causes of death in HIV-patients. Furthermore, due to the statistics even antiretroviral therapy does not have great affection on the TB and HIV coinfection course. TB/HIV coinfection is a global substantial problem and it means that all international guidelines have to be better implemented.

Keywords: HIV, TB, antiretroviral therapy, coinfection.

\section{INTRODUCTION}

TB and AIDS coinfection is one of the most dangerous states among all of the infection diseases. Furthermore, this coinfection creates a lot of difficult diagnostic and therapeutic challenges and this creates an increased pressure on health care systems. TB is top 1 cause of death in patients with AIDS (26\% of AIDS-related deaths) [2]. TB and HIV have huge effects on the host's immune system alone, however while there is a coinfection it changes the course of TB as well as AIDS because both diseases potentiate each other, that is why diagnostics as well as treatment become a very difficult task. It is known that almost the one-third of the planet population is latently infected with $\mathrm{M}$. tuberculosis, and HIV is one of the most common causes of the $M$. tuberculosis reactivation.

t is generally known that approximately one hird of the whole population are latently infected with the M. tuberculosis. Although, the rate of activation of the latent TB vary differently. However, most of the cases show that approximately $10 \%$ of latently TB-infected patients will have the disease development. Nonetheless, immunocompromised patients (HIV-positive) are 10-15\% more likely to develop the disease if the they were latently infected previously [3].
During the latent phase of the TB the bacteria is not fully eradicated so as soon as host's immune system become compromised (with HIV for example) it leads to the TB reactivation. Surely, reactivated Tb in patient with compromised immune system will lead to the changings in clinical manifestation.

In $90 \%$ of cases TB affects lungs with formation (a) which contains Mycobacteria. D4+ T cells and TNF took an importan role in granuloma organization. Thus, this explains why granuloma formation usually fails in patients with TB and AIDS coinfection. arver, Tb and AIDS coinfection lesions are much more diffuse. For example, instead of granuloma formation in the lungs, patients with TB and AIDS coinfection usually have granulocytic infiltrate with necrosis which is much more diffuse lesion in comparison with well-defined granulomas. Moreover, up to $80 \%$ patients with TB and AIDS coinfection have extra pulmonary forms of TB or even could course in a form of systemic disease Another enther phenomenon to develop is IRIS (immune reconstitution inflammatory syndrome). It could develop in patients who receive both antill still debated, however some patients have developed some of IRIS predictors including low CD4+ levels and high viral load prior to antiretroviral therapy and an increase of CD4+ counts after the beginning of the antiretroviral therapy. Possible mechanisms responsible for IRIS may be a sustained Th1-response against mycobacterial antigens, which is followed by dysregulation of cytokine secretion and $T$ cell migration to the inflammatory site. Two cypes of IRIS presentation could be described: unmasking of undiagnosed tuberculosis and a paradoxicat deterioration of existing tuberculosis lesions or appearance of new esions after initial improvement [4].

\section{DIAGNOSIS}

The most common test for the TB - Sputum smear microscopy is usually not representative in patients with $\mathrm{Tb}$ and AIDS coinfection because the rate of the smear-negative $\mathrm{Tb}$ represents up to $60 \%$ of all TB cases in HIV patients [5]. This is the main reason why the methodology of TB diagnosis in patients with 
compromised immune system have to be - One specimen from an extrapulmonary revised. With advancing immunosuppression extra pulmonary involvement, intra-thoracic mediastinal lymphadenopathy, lower lobe infiltrate and miliary TB become more common. That was the reason why WHO provided the guideline with the recommendation for the diagnostics of the TB in HIV-positive patients which helped to reduce the diagnostic delay and therefore decrease morbidity and mortality of TB in HIV-positive patients [6]

\section{SMEAR-POSITIVE PULMONARY}

- One sputum smear examination positive for acid-fast bacilli (AFB) and

- Laboratory confirmation of HIV infection or

- Strong clinical evidence of HIV infection.

Smear-negative pulmonary tuberculosis

- At least two sputum specimens negative for

AFB and

- Radiographical abnormalities consistent

with active tuberculosis and

- Laboratory confirmation of HIV infection or

- Strong clinical evidence of HIV infection and

- Decision by a clinician to treat with a ful course of antituberculosis chemotherapy

- A patient with AFB smear-negative sputum which is culture-positive for Mycobacterium tuberculosis. Extrapulmonary tuberculosis

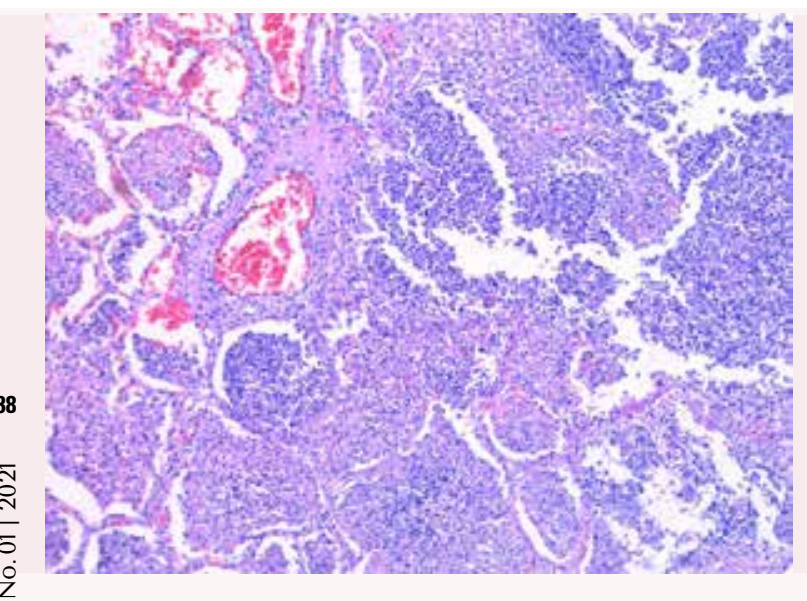

Figure 1. Caseous pneumonia in HIV infection. Staining with hematoxylin and eosin, $\mathrm{x} 100$ site culture-positive for Mycobacterium tuberculosis or smear-positive for AFB OR - Histological or strong clinical evidence consistent with active extrapulmonary tuberculosis and

- Laboratory confirmation of HIV infection or - Strong clinical evidence of HIV infection and

- A decision by a clinician to treat with a full course of antituberculosis chemotherapy. [7]

Pathomorphological examination of HIV / TB co-infection usually reveals multiple areactive caseous-necrotic and caseous-purulent foci (Figure 1) without the typical granulomatous inflammation characteristic of tuberculosi (Figure 3 and Figure 4), which often complicates the morphological verification of the tuberculosis process (Figure 1). In the study of micropreparations stained by the method of purulent masses can be found a large number of acid-fast mycobacteria (Figure 2).

\section{TREATMENT}

The TB and HIV coinfection treatment is stil a very discussable problem. Multiple studie were launched, however there are still no clea evidences for some special treatment for such type of patients, so they should receive the

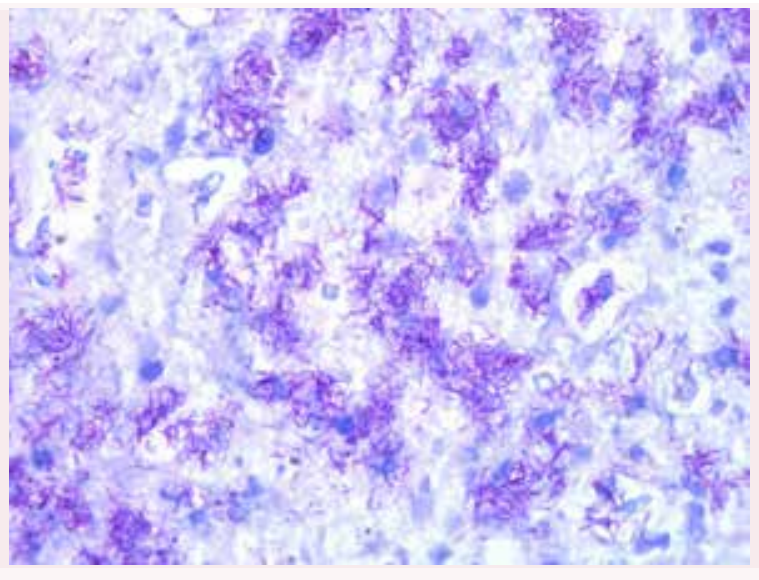
berculosis in the foci of caseous pneumonia in HIV infection. Stained with Ziehl-Neelsen stain, x1000. Ziel-Nielsen, in caseous-necrotic and caseous-

Figure 2. Acid-resistant mycobacteria of tu-

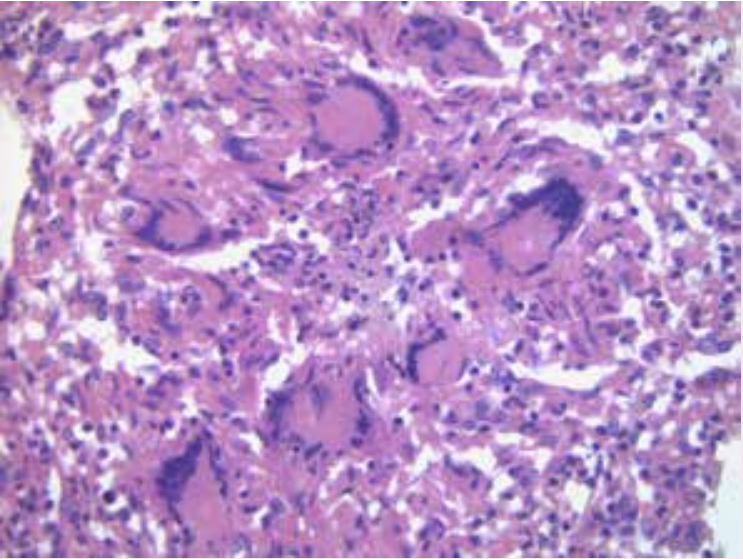

Figure 3. Non-necrotizing giant cell granulomas in lymph node tuberculosis without HIV infection. Staining with hematoxylin and eo$\sin , x 400$

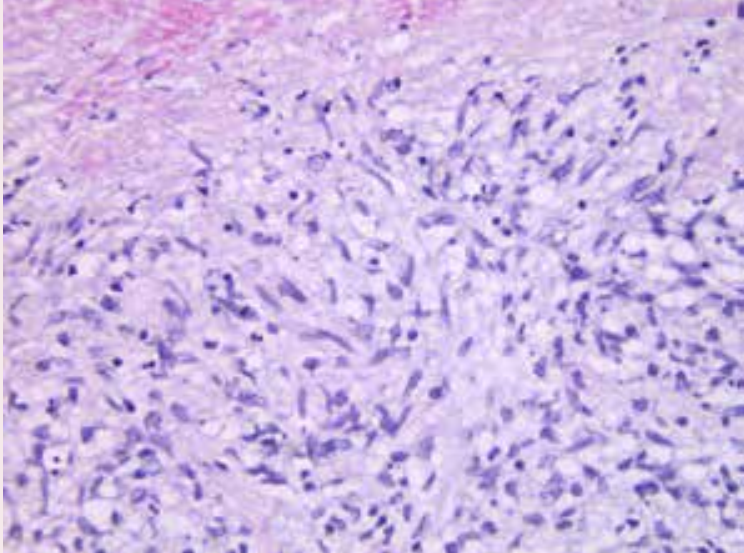

Figure 4. Epithelioid granulomas in lymph node tuberculosis without HIV infection Staining with hematoxylin and eosin, $x 400$

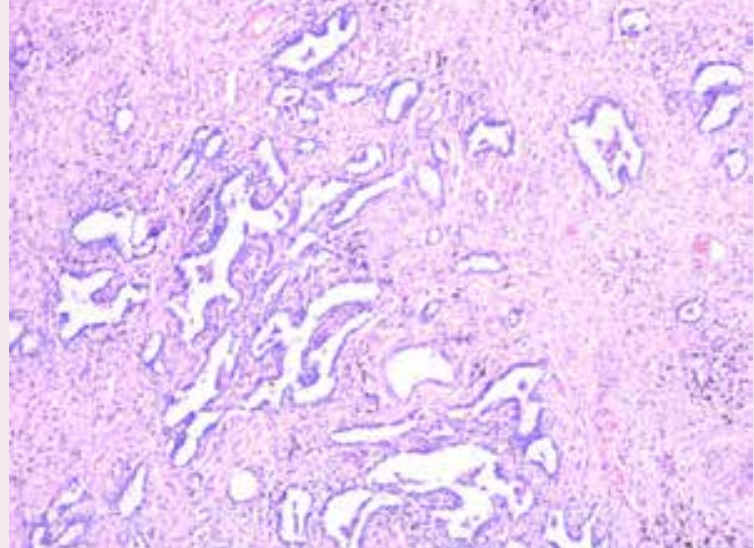

Figure 5. Remodeling of the lung parenchyma in the healing of tuberculous inflammation. Staining with hematoxylin and eosin $\mathrm{x} 100$.

treatment for both HIV and Tb respectively treatment of the TB and AIDS coinfection the $[8,9]$. One of the main tactics is to begin numbers of patients with this coinfection still ART within the first few weeks as soon as relatively huge, thus global healthcare systems TB treatment is tolerated and the patient is have to conduct new researches in this field to stable, after treatment of active opportunistic lower the morbidity and mortality rates. infections, especially for patients with profound immunosuppression [4]. However, the timing of the beginning of ART therapy is still a very discussable question.

\section{CONCLUSION:}

TB and HIV coinfection remains a huge problem for a global healthcare system. Despite all the huge improvements in diagnosis and 


\section{REFERENCES}

1. MacNeil A, Glaziou P, Sismanidis C, Date A, Maloney S, Floyd K. Global Epidemiology of Tuberculosis and Progress Toward Meeting Global Targets - Worldwide, 2018. MMWR Morb Mortal Wkly Rep 2020;69:281-285.

2. World Health Organization. Global tuberculosis report 2019. Geneva, Switzerland: World Health Organization; 2019.

3. CDC. The Difference Between Latent TB Infection and TB Disease / CDC. - 2020.

4. Swaminathan S. Diagnosis \& treatment of tuberculosis in HIV co-infected patients / S. Swaminathan, C. Padmapriyadarsini. // Indian journal of medical research. 2011. - C. 850-865.

5. WHO. Tuberculosis and HIV. WHO // WHO. - 2020.

6. Abebe $G$, Deribew A, Apers $L$, Abdissa A, Kiflie $Y$, Koole $O$, Colebunders R. Evaluation of the $2007 \mathrm{WHO}$ guideline to diagnose smear negative tuberculosis in an urban hospital in Ethiopia. BMC Infect Dis. 2013 Sep 11;13:427. doi: 10.1186/1471-2334-13-427. PMID: 24020936; PMCID: PMC3849989.
7. World Health Organization. Improving the diagnosis and treatment of smearnegative pulmonary and extrapulmonary tuberculosis among adults and adolescents Recommendations for HIV-prevalent and resource-constrained settings. 2007. Geneva, Switzerland: World Health Organization; 2007

8. Immune reconstitution inflammatory syndrome in HIV-infected patients Naomi F Walker, James Scriven, Graeme Meintjes, Robert J Wilkinson HIV AIDS (Auckl) 2015; 7: $49-64$.

9. Faiz A. Khan, Jessica Minion, Madhukar Pai, Sarah Royce, William Burman, Anthony D. Harries, Dick Menzies, Treatment of Active Tuberculosis in HIV-Coinfected Patients: A Systematic Review and MetaAnalysis, Clinical Infectious Diseases, Volume 50, Issue 9, 1 May 2010, Pages $1288-1299$

Corresponding author: Andriy Yu. Horoshchak, a.horoshchak@kmu.edu.ua

Manuscript was recieved on 25 October; accepted for publication on 26 December.

$\begin{array}{lccc}\text { CITE AS: PETRO V. KUZYK ET } & \text { AL. } \\ \text { MORPHOLOGICAL } & \text { FEATURES } & \text { OF } \\ \text { SECONDARY LUNG } & \text { TUBERCULOSIS } & \text { IN } \\ \text { HIV-PATIENTS } & & \end{array}$

BIOMEDICAL UPDATE, ISSUE 1, 2021 